PDES, SUBMANIFOLDS AND

AFFINE DIFFERENTIAL GEOMETRY

BANACH CENTER PUBLICATIONS, VOLUME 57

INSTITUTE OF MATHEMATICS

POLISH ACADEMY OF SCIENCES

WARSZAWA 2002

\title{
CR SUBMANIFOLDS OF MAXIMAL CR DIMENSION IN COMPLEX MANIFOLDS
}

\author{
MIRJANA DJORIĆ \\ Faculty of Mathematics, University of Belgrade \\ Studentski trg 16, pb 550, 11000 Belgrade, Yugoslavia \\ E-mail:mdjoric@matf.bg.ac.yu \\ MASAFUMI OKUMURA \\ Department of Mathematics, Saitama University \\ Shimo-Okubo, Urawa, 338 Japan \\ E-mail: mokumura@rimath.saitama-u.ac.jp
}

\begin{abstract}
The aim of this paper is to investigate $n$-dimensional real submanifolds of complex manifolds in the case when the maximal holomorphic tangent space is $(n-1)$-dimensional. In particular, we give some examples and we consider the Levi form on these submanifolds, especially when the ambient space is a complex space form. Moreover, we show that on some remarkable class of real hypersurfaces of complex space forms, the Levi form cannot vanish identically.
\end{abstract}

0. Introduction. Let $M$ be a real submanifold of a complex manifold $\bar{M}$ and let $J$ be the complex structure of $\bar{M}$. If the maximal $J$-invariant tangent subspace of $M$, called holomorphic tangent space, has constant dimension over $M$, then $M$ is called a Cauchy-Riemann submanifold, or briefly a CR submanifold, and the constant complex dimension is called the CR dimension of $M([10])$. This paper is devoted to the study of such submanifolds. In section 1 we give an example in which this dimension is not constant. Moreover, we prove that the above-given definition of CR submanifolds is not always equivalent to a well-known definition of Bejancu ([1]), although they coincide in the case when at each point the real dimension of the holomorphic tangent space is $n-1$. In section 2 we prove that the complexification of the holomorphic tangent space is not involutive and we use the notion of the Levi form, which measures the degree to which this space fails to be involutive.

2000 Mathematics Subject Classification: 53C15, 53C25, 53C40.

Key words and phrases: CR submanifold, holomorphic tangent space, real submanifold, complex space form, Levi form.

The paper is in final form and no version of it will be published elsewhere. 
It is well-known that a real hypersurface is one of the typical examples of CR submanifolds and its CR dimension is $\frac{n-1}{2}$, where $n$ is the dimension of a hypersurface. In the case of a real hypersurface, and in particular when $\bar{M}$ is a complex space form, many results are known. We refer to [5], [6], [7], [9], [11] for more details and further references. The main purpose of this paper is to investigate the case when $M$ is a $\mathrm{CR}$ submanifold with arbitrary codimension, whose CR dimension is $\frac{n-1}{2}$. In section 3 we recall some general preliminary facts concerning these submanifolds and we give some examples. Finally, in section 4 we consider CR submanifolds of complex space forms and we prove our main results.

The authors are grateful to the organizers of the meeting in Warsaw for their hospitality and support.

1. The holomorphic tangent space. Let $M$ be an $n$-dimensional real submanifold of codimension $p$ of a complex manifold $(\bar{M}, J)$, where $J$ is the almost complex structure of $\bar{M}$. The subspace $H_{x}(M)=J T_{x}(M) \cap T_{x}(M)$ is the maximal $J$-invariant subspace of the tangent space $T_{x}(M)$ at $x$ and it is called the holomorphic tangent space to $M$ at $x$. It is an even-dimensional subspace of $T_{x}(M)$, since $J$ restricted to the holomorphic subspace satisfies $J^{2}=-i d e n t i t y$. The totally real part of the tangent space of $M$ is the quotient space $R_{x}(M)=T_{x}(M) / H_{x}(M)$. We note that $J R_{x}(M) \cap R_{x}(M)=\{0\}$, since $H_{x}(M)$ is the maximal $J$-invariant subspace of $T_{x}(M)$. Therefore, $T_{x}(M)=H_{x}(M) \oplus R_{x}(M)$.

Lemma 1.1. Let $M$ be an $n$-dimensional real submanifold of a real $n+p$-dimensional complex manifold $\bar{M}$. Then, $n-p \leq \operatorname{dim}_{\mathbf{R}} H_{x}(M) \leq n$.

Proof. Since $H_{x}(M)$ is a subspace of $T_{x}(M)$, it follows $\operatorname{dim}_{\mathbf{R}} H_{x}(M) \leq \operatorname{dim} T_{x}(M)=$ $n$. To establish the other inequality, we note that $T_{x}(\bar{M}) \supset T_{x}(M)+J T_{x}(M)$ and therefore

$$
\operatorname{dim}_{\mathbf{R}} T_{x}(\bar{M}) \geq \operatorname{dim} T_{x}(M)+\operatorname{dim} J T_{x}(M)-\operatorname{dim}_{\mathbf{R}} H_{x}(M) .
$$

Since $\operatorname{dim} J T_{x}(M)=\operatorname{dim} T_{x}(M)=n$, it follows that $\operatorname{dim}_{\mathbf{R}} H_{x}(M) \geq n-p$.

The lemma states that $\operatorname{dim}_{\mathbf{R}} H_{x}(M)$ is an even number between $n-p$ and $n$. If $M$ is a real hypersurface, then $p=1$ and the only possibility is $\operatorname{dim}_{\mathbf{R}} H_{x}(M)=n-1$, i.e. the dimension of $H_{x}(M)$ is constant. However, there are more possibilities for $p>1$.

EXAmple 1.1. Let $M=\left\{z=\left(z_{1}, \ldots, z_{n}\right) \in \mathbf{C}^{n} ;|z|=1, \operatorname{Im} z_{n}=0\right\}$, i.e. $M$ is just the equator of the unit sphere in $\mathbf{C}^{n}$. Here $\operatorname{dim} M=2 n-2, p=2$ and therefore $2 n-4 \leq$ $\operatorname{dim}_{\mathbf{R}} H_{x}(M) \leq 2 n-2$, for $x \in M$. At the point $p_{1}=\left(z_{1}=0, \ldots, z_{n-2}=0, z_{n-1}=\right.$ $\left.1, z_{n}=0\right\} \in M$, tangent space $T_{p_{1}}(M)$ is spanned over $\mathbf{R}$ by $\left\{\partial / \partial x_{1}, \partial / \partial y_{1}, \ldots, \partial / \partial x_{n-2}\right.$, $\left.\partial / \partial y_{n-2}, \partial / \partial y_{n-1}, \partial / \partial x_{n}\right\}$. The vectors $J\left(\partial / \partial x_{n}\right)=\partial / \partial y_{n}$ and $J\left(\partial / \partial y_{n-1}\right)=-\left(\partial / \partial x_{n-1}\right)$ are orthogonal to $T_{p_{1}}(M)$ and therefore $\partial / \partial y_{n-1}$ and $\partial / \partial x_{n}$ span $R_{p_{1}}(M)$. The vectors $\left\{\partial / \partial x_{1}, \partial / \partial y_{1}, \ldots, \partial / \partial x_{n-2}, \partial / \partial y_{n-2}\right\}$ span the $J$-invariant subspace $H_{p_{1}}(M)$. Therefore, in this case, $\operatorname{dim}_{\mathbf{R}} H_{p_{1}}(M)=2 n-4$.

Further, let us consider the point $p_{2}=\left(z_{1}=0, \ldots, z_{n-1}=0, z_{n}=1\right) \in M$. Here, $T_{p_{2}}(M)$ is spanned by $\left\{\partial / \partial x_{1}, \partial / y_{1}, \ldots, \partial / \partial x_{n-1}, \partial / \partial y_{n-1}\right\}$ which is $J$-invariant. Therefore, $H_{p_{2}}(M)=T_{p_{2}}(M)$ and $\operatorname{dim}_{\mathbf{R}} H_{p_{2}}(M)=2 n-2$. 
Since in the above example the dimension of $H_{x}(M)$ varies with $x$, we recall the following definition.

Definition 1.1 ([10]). If the subspace $H_{x}(M)$ has constant dimension for $x \in M$, the submanifold $M$ is called the Cauchy-Riemann submanifold or briefly CR submanifold and the constant complex dimension of $H_{x}(M)$ is called the $C R$ dimension of $M$.

However, Bejancu gave another definition of a CR submanifold of an almost Hermitian manifold $(\bar{M}, \bar{g}, J)$ :

Definition 1.2 ([1]). A submanifold $M$ of $(\bar{M}, J)$ is called a $C R$ submanifold if it is endowed with a pair of mutually orthogonal and complementary distributions $\left(\Delta, \Delta^{\perp}\right)$ such that for any $x \in M$ we have $J \Delta_{x}=\Delta_{x}$ and $J \Delta_{x}^{\perp} \subset T_{x}^{\perp}(M)$.

It is easily seen that if $M$ is a CR submanifold in the sense of Bejancu, $M$ is also a CR submanifold in the sense of Definition 1.1. In the case when $M$ is a CR submanifold of CR dimension $\frac{n-1}{2}$, the definition by Bejancu coincides with the Definition 1.1 and $\operatorname{dim} \Delta^{\perp}=1$. On the other hand, as we show below, when the CR dimension is less than $\frac{n-1}{2}$, the converse is wrong.

Let $M^{n}$ be a submanifold of $(\bar{M}, \bar{g}, J)$ which is a CR submanifold of CR dimension $\frac{n-2}{2}$. Choosing an orthonormal basis $e_{1}, e_{2}, \ldots, e_{n-2}, e_{n-1}, e_{n}$ of $T_{x}(M)$ in such a way that $e_{1}, e_{2}, \ldots, e_{n-2} \in J T_{x}(M) \cap T_{x}(M)$, we have

$$
\begin{aligned}
& J e_{i} \in J T_{x}(M) \cap T_{x}(M), \quad(i=1,2, \ldots, n-2), \\
& J e_{n-1}=\sum_{i=1}^{n-2} a^{i} e_{i}+\lambda e_{n}+\text { normal part, } \\
& J e_{n}=\sum_{i=1}^{n-2} b^{i} e_{i}-\lambda e_{n-1}+\text { normal part }
\end{aligned}
$$

and it follows that $a^{i}=0$ and $b^{i}=0$ for $i=1, \ldots, n-2$ and that $\lambda=\bar{g}\left(J e_{n-1}, e_{n}\right)$. Choosing other orthonormal vectors, $e_{n-1}^{\prime}$ and $e_{n}^{\prime}$, we have

$$
\begin{aligned}
e_{n-1}^{\prime} & =e_{n-1} \cos \theta+e_{n} \sin \theta, \\
e_{n}^{\prime} & =-e_{n-1} \sin \theta+e_{n} \cos \theta,
\end{aligned}
$$

for some $\theta$, and therefore

$$
\lambda^{\prime}=\bar{g}\left(J e_{n-1}^{\prime}, e_{n}^{\prime}\right)=\lambda \bar{g}\left(e_{n} \cos \theta-e_{n-1} \sin \theta,-e_{n-1} \sin \theta+e_{n} \cos \theta\right)=\lambda .
$$

This shows that $\lambda$ is independent of the choice of $e_{n-1}$ and $e_{n}$. In particular, let $M=$ $\left\{\left(z_{1}, z_{2}\right) \in \mathbf{C}^{2} \mid \operatorname{Im} z_{1}=\operatorname{Re} z_{2}, \operatorname{Im} z_{2}=0\right\}$, that is, $M=\left\{\left(x_{1}, y_{1}, y_{1}, 0\right) \in \mathbf{R}^{4} \mid x_{1}, y_{1} \in \mathbf{R}\right\}$. Then $M$ is totally real and $\operatorname{dim} H(M)=0$. If we choose $e_{1}=\frac{\partial}{\partial x_{1}}, e_{2}=\frac{\partial}{\partial y_{1}}+\frac{\partial}{\partial x_{2}}, f_{1}=$ $\frac{\partial}{\partial y_{1}}-\frac{\partial}{\partial x_{2}}, f_{2}=\frac{\partial}{\partial y_{2}}$, it follows that $e_{1}, e_{2} \operatorname{span} T(M)$ and that $f_{1}, f_{2} \operatorname{span} T^{\perp}(M)$. From $J\left(\partial / \partial x_{i}\right)=\partial / \partial y_{i}$ and $J\left(\partial / \partial y_{i}\right)=-\partial / \partial x_{i}$, it follows $\lambda \neq 0$. Thus the CR submanifold in the sense of Definition 1.1 is not always a CR submanifold in the sense of Definition 1.2.

2. The Levi form. In the following, we assume that $M$ is a real submanifold of a complex manifold $(\bar{M}, J)$, with the immersion $f$ of $M$ into $\bar{M}$ (we denote also by $f$ the differential of the immersion), and whose holomorphic tangent space at $x$ is $H_{x}(M)$. 
Further, let $H_{x}^{C}(M)$ be the complexification of $H_{x}(M)$ and

$$
\begin{aligned}
& H_{x}^{(0,1)}(M)=\left\{f X+\sqrt{-1} J f X \mid X \in H_{x}(M)\right\}, \\
& H_{x}^{(1,0)}(M)=\left\{f X-\sqrt{-1} J f X \mid X \in H_{x}(M)\right\} .
\end{aligned}
$$

Then we have $H_{x}^{C}(M)=H_{x}^{(0,1)}(M) \oplus H_{x}^{(1,0)}(M)$. We define the following subbundle of the complexification of the tangent bundle $T^{C}(M)$ :

$$
H^{C}(M)=\bigcup_{x \in M} H_{x}^{C}(M), H^{(0,1)}(M)=\bigcup_{x \in M} H_{x}^{(0,1)}(M), H^{(1,0)}(M)=\bigcup_{x \in M} H_{x}^{(1,0)}(M) .
$$

Then we have

Proposition 2.1. Both distributions $H^{(0,1)}(M)$ and $H^{(1,0)}(M)$ are involutive.

Proof. We only show that $H^{(0,1)}(M)$ is involutive, because the other case can be proved in entirely the same way. Let $V, W \in H^{(0,1)}(M)$. Then, for some $X, Y \in H(M)$,

$$
\begin{aligned}
{[V, W] } & =[f X+\sqrt{-1} J f X, f Y+\sqrt{-1} J f Y] \\
& =[f X, f Y]-[J f X, J f Y]+\sqrt{-1}([J f X, f Y]+[f X, J f Y]) .
\end{aligned}
$$

Since $\bar{M}$ is a complex manifold, the Nijenhuis tensor

$$
\bar{N}(f X, f Y)=J[f X, f Y]-J[J f X, J f Y]-[f X, J f Y]-[J f X, f Y]
$$

vanishes identically. Hence we have

$$
\begin{aligned}
& {[f X, f Y]-[J f X, J f Y]=J(-[f X, J f Y]-[J f X, f Y]),} \\
& {[f X, J f Y]+[J f X, f Y]=J([f X, f Y]-[J f X, J f Y])}
\end{aligned}
$$

from which it follows

$$
[V, W]=[f X, f Y]-[J f X, J f Y]+\sqrt{-1} J([f X, f Y]-[J f X, J f Y]) .
$$

Since $X, Y \in H(M)$, then $J f X, J f Y \in T(M)$. Hence we may write that $J f X=f F X$ and $J f Y=f F Y$, where $F$ is a skew-symmetric endomorphism acting on $T(M)$. Thus we have $[f X, f Y]-[J f X, J f Y] \in T(M)$. Also from the above discussions, $[f X, f Y]-$ $[J f X, J f Y] \in J T(M)$, which implies that $H^{(0,1)}(M)$ is involutive.

Further, using relation (2.1), we get

$$
f[X, Y]-f[F X, F Y]=J([J f Y, f X]-[J f X, f Y]) .
$$

Since the left-hand side members of the last equation are tangent to $M$, the normal part of the right-hand side members must be zero and we obtain

Lemma 2.1. The normal part of $J[J f X, f Y]$ is equal to the normal part of $J[J f Y, f X]$.

As we have shown, both $H^{(0,1)}(M)$ and $H^{(1,0)}(M)$ are involutive. However, this does not imply that $H^{C}(M)=H^{(0,1)}(M) \oplus H^{(1,0)}(M)$ is involutive. Now we consider the involutivity of $H^{C}(M)$.

Let $V \in H^{(0,1)}(M), W \in H^{(1,0)}(M)$. Then, $V=f X+\sqrt{-1} J f X$ and $W=f Y-$ $\sqrt{-1} J f Y$, for some $X, Y \in H(M)$, and consequently

$$
[V, W]=[f X, f Y]+[J f X, J f Y]+\sqrt{-1}([f X, J f Y]-[J f X, f Y]) .
$$


Since $X, Y \in H(M)$, it follows $J f=f F X, J f Y=f F Y$ and $[J f X, J f Y],[f X, J f Y]$, $[J f X, f Y] \in T(M)$. Hence $[V, W] \in T^{C}(M)$. However, in general, $[V, W] \notin J T^{C}(M)$.

The Levi form is defined in such a way that it measures the degree to which $H^{C}(M)$ fails to be involutive. For more details, see [4], [7].

Definition 2.1. The Levi form $L$ is the projection of $J[J f X, f Y]$ to $T(M)^{\perp}$ for $X$, $Y \in H(M)$.

Lemma 2.2. A necessary and sufficient condition for $[V, W] \in H^{C}(M)$ is $J[f X, J f Y]-$ $J[J f X, f Y] \in T(M)$.

Proof. First, we note that $J[f X, J f Y]-J[J f X, f Y] \in T(M)$ is equivalent to $[f X, J f Y]-[J f X, f Y] \in J T(M)$. Then by the definition of $H^{C}(M)$, the necessity is trivial. To prove the sufficiency, we take $X^{\prime} \in H(M)$. Then $X^{\prime} \in J(T(M))$ implies that there exists $X \in T(M)$ such that $f X^{\prime}=J f X$ and we have

$\left[f X^{\prime}, f Y\right]+\left[J f X^{\prime}, J f Y\right]=[J f X, f Y]+\left[J^{2} f X, J f Y\right]=[J f X, f Y]-[f X, J f Y] \in J T(M)$, which completes the proof.

Thus, we know that $H^{C}(M)$ is involutive if and only if the Levi form vanishes identically.

THEOREM 2.1 ([4]). Let $\bar{M}$ be a complex manifold with torsion-free affine connection $\bar{\nabla}$ whose parallel translation leaves the almost complex structure $J$ invariant and let $M$ be a real submanifold of $\bar{M}$. Then we have

$$
L(X, Y)=h(X, Y)+h(F X, F Y),
$$

for $X, Y \in H(M)$, where $h$ denotes the second fundamental form with respect to $\bar{\nabla}$.

Proof. We compute

$$
\begin{aligned}
J[J f X, f Y] & =J\left(\bar{\nabla}_{J f X} f Y-\bar{\nabla}_{f Y}(J f X)\right)=J\left(\bar{\nabla}_{f F X} f Y-J \bar{\nabla}_{f Y} f X\right) \\
& =\bar{\nabla}_{f F X} J f Y+\bar{\nabla}_{f Y} f X=\bar{\nabla}_{f F X} f F Y+\bar{\nabla}_{f Y} f X \\
& =f \nabla_{F X}(F Y)+h(F X, F Y)+f \nabla_{Y} X+h(Y, X),
\end{aligned}
$$

which proves the theorem.

3. CR submanifold of maximal CR dimension. Let $M$ be an $n$-dimensional real submanifold of codimension $p$ of a Hermitian manifold $\bar{M}$ with structure tensor field $J$ and Hermitian metric $\bar{g}$, where $n>1$. Also, we denote by $f$ the immersion of $M$ into $\bar{M}$. Then the tangent bundle $T(M)$ is identified with a subbundle of $T(\bar{M})$ and a Riemannian metric $g$ of $M$ is induced from the Riemannian metric $\bar{g}$ of $\bar{M}$ in such a way that $g(X, Y)=\bar{g}(f X, f Y)$ where $X, Y \in T(M)$ and we denote also by $f$ the differential of the immersion. However, sometimes we omit to mention $f$ for brevity of notation. The normal bundle $T^{\perp}(M)$ is the subbundle of $T(\bar{M})$ consisting of all $\bar{X} \in T(\bar{M})$ which are orthogonal to $T(M)$ with respect to the Riemannian metric $\bar{g}$.

Now, let $M$ be a CR submanifold of maximal CR dimension, that is, at each point $x$ of $M$, let the real dimension of $J T_{x}(M) \cap T_{x}(M)$ be $n-1$. Then $M$ is necessarily odd-dimensional and there exists a unit vector $\xi_{x}$ normal to $T_{x}(M)$ such that $J T_{x}(M) \subset$ 
$T_{x}(M) \oplus \operatorname{span}\left\{\xi_{x}\right\}$ for any $x \in M$. Hence, for any $X \in T(M)$, we may write

$$
J f X=f F X+u(X) \xi,
$$

where $F$ defines a skew-symmetric linear transformation of the tangent bundle $T(M)$ of $M$, while $u$ defines a 1-form on a neighborhood of $x \in M$. Let $\eta$ be an element of $T^{\perp}(M)$ which is orthogonal to $\xi$, then $\bar{g}(J \eta, f X)=-\bar{g}(\eta, J f X)=0$. On the other hand, the Hermitian property of $\bar{g}$ and $J$ implies that

$$
0=\bar{g}(f X, \eta)=\bar{g}(J f X, J \eta)=\bar{g}(f F X, J \eta)+u(X) \bar{g}(\xi, J \eta)=u(X) \bar{g}(\xi, J \eta) .
$$

If at a point $x \in M$, we have $u_{x}(X)=0$ for any $X \in T(M)$, then from (3.1) it follows that $T_{x}(M)$ is $J$-invariant and, consequently, even-dimensional, which is a contradiction. Therefore we have $\bar{g}(\xi, J \eta)=0$. This means that $J \eta \perp T(M) \oplus \operatorname{span}\{\xi\}$. In other words, the subbundle $T_{1}^{\perp}(M)=\left\{\eta \in T^{\perp}(M) \mid \bar{g}(\eta, \xi)=0\right\}$ is $J$-invariant, from which we have

$$
J \xi=-f U \text {, }
$$

because $\bar{g}(J \xi, \eta)=-\bar{g}(\xi, J \eta)=0$.

Further, in $T^{\perp}(M)$ we choose a local orthonormal basis $\xi, \xi_{1}, \ldots, \xi_{q}, \xi_{1^{*}}, \ldots, \xi_{q^{*}}$, where $\xi_{a^{*}}=J \xi_{a}, a=1, \ldots, q$ and $q=\frac{p-1}{2}$.

Applying $J$ to (3.1), (3.2) and comparing the tangential parts and the normal parts to $M$, we have

$$
\begin{gathered}
F^{2} X=-X+u(X) U, \\
u(F X)=0, \quad F U=0, \\
g(U, X)=u(X),
\end{gathered}
$$

where $g$ denotes the induced Riemannian metric from $\bar{g}$ to $M$.

We denote by $\bar{\nabla}$ and $\nabla$ the Riemannian connections of $\bar{M}$ and $M$ respectively and by $D$ the normal connection induced from $\bar{\nabla}$ in the normal bundle $T^{\perp}(M)$. Then we have

$$
\bar{\nabla}_{f X} f Y=f \nabla_{X} Y+h(X, Y),
$$

The Weingarten equations are the following:

$$
\begin{gathered}
\bar{\nabla}_{f X} \xi=-f A X+D_{X} \xi=-f A X+\sum_{a=1}^{q}\left\{s_{a}(X) \xi_{a}+s_{a^{*}}(X) \xi_{a^{*}}\right\} \\
\bar{\nabla}_{f X} \xi_{a}=-f A_{a} X+D_{X} \xi_{a}=-f A_{a} X-s_{a}(X) \xi+\sum_{b=1}^{q}\left\{s_{a b}(X) \xi_{b}+s_{a b^{*}}(X) \xi_{b^{*}}\right\} \\
\bar{\nabla}_{f X} \xi_{a^{*}}=-f A_{a^{*}} X+D_{X} \xi_{a^{*}}=-f A_{a^{*}} X-s_{a^{*}}(X) \xi+\sum_{b=1}^{q}\left\{s_{a^{*} b}(X) \xi_{b}+s_{a^{*} b^{*}}(X) \xi_{b^{*}}\right\}
\end{gathered}
$$

where $A, A_{a}, A_{a^{*}}$ are the shape operators for the normals $\xi, \xi_{a}, \xi_{a^{*}}$ respectively and $s^{\prime}$ s are the coefficients of the normal connection $D$.

When the ambient complex manifold is a Kaehler manifold, then $\bar{\nabla} J=0$. Since $\xi_{a^{*}}=J \xi_{a}$, this, together with (3.8) and (3.9), implies that

$$
\begin{gathered}
A_{a^{*}} X=F A_{a} X-s_{a}(X) U, \\
s_{a^{*}}(X)=u\left(A_{a} X\right)=g\left(A_{a} X, U\right)=g\left(A_{a} U, X\right),
\end{gathered}
$$




$$
s_{a^{*} b^{*}}=s_{a b}, s_{a^{*} b}=s_{a b^{*}} .
$$

Also, from (3.6), (3.7), (3.8) and (3.9), it follows that

$$
h(X, Y)=g(A X, Y) \xi+\sum_{a=1}^{q}\left\{g\left(A_{a} X, Y\right) \xi_{a}+g\left(A_{a^{*}} X, Y\right) \xi_{a^{*}}\right\} .
$$

Here we give some examples of CR submanifolds of maximal CR dimension.

EXAMPLE 3.1. In the case when $M$ is a real hypersurface of an almost Hermitian manifold $\bar{M}$, the maximal $J$-invariant subspace is necessarily $(n-1)$-dimensional and when the ambient subspace $\bar{M}$ is a complex manifold, it is the maximal holomorphic subspace. Therefore, real hypersurfaces of complex manifolds are CR submanifolds of maximal CR dimension.

EXAMPLE 3.2. Let $M^{\prime}$ be a complex submanifold of $\bar{M}$ with immersion $f_{1}$ and $M$ a real hypersurface of $M^{\prime}$ with immersion $f_{0}$ and $f=f_{1} f_{0}$. We denote by $\xi^{\prime}$ the unit normal vector field to $M$ in $M^{\prime}$. Since $f_{1}$ is holomorphic, it follows that $f_{1} J^{\prime}=J f_{1}$, where $J^{\prime}$ is the induced almost complex structure of $M^{\prime}$ from $J$. Now we have, for $X \in T(M)$,

$$
J f X=J f_{1} f_{0} X=f_{1} J^{\prime} f_{0} X=f_{1}\left(f_{0} F^{\prime} X+u(X) \xi^{\prime}\right)=f F^{\prime} X+u(X) f_{1} \xi^{\prime} .
$$

On the other hand, we may write

$$
J f X=f F X+\sum_{a=1}^{p} u^{a}(X) \xi_{a}
$$

where $\xi_{a}, a=1, \ldots, p$ are local orthonormal vector fields normal to $M$ in $\bar{M}$. We choose $\xi$ in such a way that $\xi=f_{1} \xi^{\prime}$, then $J f X=f F X+u(X) \xi$. Thus, any real hypersurface $M$ of a complex submanifold $M^{\prime}$ of $\bar{M}$ is a CR submanifold with maximal CR dimension.

EXAMPLE 3.3. Let $M^{\prime}$ be a real hypersurface of $\bar{M}$ and let $f_{1}$ be the immersion. Then, for any $X^{\prime} \in T\left(M^{\prime}\right)$, we put

$$
J f_{1} X^{\prime}=f_{1} F^{\prime} X+u^{\prime}\left(X^{\prime}\right) \xi .
$$

In this case $F^{\prime}, u^{\prime}$ and the induced Riemannian metric $g^{\prime}$ from the Hermitian metric of $\bar{M}$ define an almost contact metric structure of $M^{\prime}$.

Let $M$ be an $F^{\prime}$-invariant submanifold of $M^{\prime}$, that is, $M$ satisfies $F^{\prime} T(M) \subset T(M)$. Denote by $f_{0}$ the immersion and put $f=f_{1} f_{0}$. We choose a local orthonormal basis of $T^{\perp}(M)$ in $T(\bar{M})$ in such a way that $\xi_{1}=\xi$ and $\xi_{2}, \ldots, \xi_{p}$ are orthonormal in $T\left(M^{\prime}\right)$. Then, for $X \in T(M)$,

$$
J f X=f F X+\sum_{a=1}^{p} u^{a}(X) \xi_{a}
$$

Also, we have

$$
J f X=J f_{1} f_{0} X=f_{1} F^{\prime} f_{0} X+u^{\prime}\left(f_{0} X\right) \xi=f_{1} f_{0} F X+u^{\prime}\left(f_{0} X\right) \xi=f F X+u^{\prime}\left(f_{0} X\right) \xi,
$$

because $M$ is $F^{\prime}$-invariant submanifold. Comparing the above two equations, we have $u^{1}(X)=u^{\prime}\left(f_{0} X\right), u^{a}(X)=0, a=2, \ldots, p$. Hence any $F^{\prime}$-invariant submanifold of a real hypersurface of $\bar{M}$ is a $\mathrm{CR}$ submanifold of maximal $\mathrm{CR}$ dimension. 
In the following, we assume that the normal vector field $\xi$ is parallel with respect to the normal connection $D$ which is induced from $\bar{\nabla}$. Then,

$$
D_{X} \xi=\sum_{a=1}^{q}\left\{s_{a}(X) \xi_{a}+s_{a^{*}}(X) \xi_{a^{*}}\right\}=0,
$$

from which it follows

$$
s_{a}=s_{a^{*}}=0, \quad a=1,2, \ldots, q,
$$

and, from (3.10) and (3.11),

$$
\begin{aligned}
A_{a^{*}} & =F A_{a}, \quad a=1,2, \ldots, q, \\
A_{a} U & =0, \quad a=1,2, \ldots, q .
\end{aligned}
$$

4. Real submanifolds of a complex space form. Let $\bar{M}$ be a Kaehler manifold. Then the Riemannian connection $\bar{\nabla}$ leaves the almost complex structure $J$ invariant, that is, $\bar{\nabla} J=0$. We differentiate (3.1) and (3.2) covariantly and compare the tangential parts and the normal parts. Then we obtain

$$
\begin{gathered}
\left(\nabla_{X} F\right) Y=u(Y) A X-g(A Y, X) U, \\
\left(\nabla_{Y} u\right)(X)=g(F A Y, X), \\
\nabla_{X} U=F A X .
\end{gathered}
$$

We assume that the ambient manifold $\bar{M}$ is a complex space form, that is, a Kaehler manifold of constant holomorphic sectional curvature $4 k$. Then the curvature tensor $\bar{R}$ of $M$ has the form of

$$
\bar{R}(\bar{X}, \bar{Y}) \bar{Z}=k\{\bar{g}(\bar{Y}, \bar{Z}) \bar{X}-\bar{g}(\bar{X}, \bar{Z}) \bar{Y}+\bar{g}(J \bar{Y}, \bar{Z}) J \bar{X}-\bar{g}(J \bar{X}, \bar{Z}) J \bar{Y}-2 \bar{g}(J \bar{X}, \bar{Y}) J \bar{Z}\}
$$

for $\bar{X}, \bar{Y}, \bar{Z} \in T(\bar{M})$. Consequently, using (3.14) and (3.15), the Gauss equation and the Codazzi equation for the normal $\xi$ become respectively

$$
\begin{aligned}
R(X, Y) Z= & k\{g(Y, Z) X-g(X, Z) Y+g(F Y, Z) F X-g(F X, Z) F Y \\
& -2 g(F X, Y) F Z\}+g(A Y, Z) A X-g(A X, Z) A Y \\
& +\sum_{a=1}^{q}\left\{g\left(A_{a} Y, Z\right) A_{a} X-g\left(A_{a} X, Z\right) A_{a} Y\right. \\
& \left.+g\left(F A_{a} Y, Z\right) F A_{a} X-g\left(F A_{a} X, Z\right) F A_{a} Y\right\} \\
\left(\nabla_{Y} A\right) X- & \left(\nabla_{X} A\right) Y=k\{u(Y) F X-u(X) F Y+2 g(F X, Y) U\}
\end{aligned}
$$

for $X, Y, Z \in T(M)$, where $R$ denotes the curvature tensor of $M$.

In the following, we establish several formulas in the case when $U$ is the eigenvector of the shape operator $A$. Let $U$ be an eigenvector of $A$ corresponding to the eigenvalue $\alpha$. Taking the covariant derivative of $A U=\alpha U$ and using (4.3), we obtain

$$
\left(\nabla_{X} A\right) U+A F A X=(X \alpha) U+\alpha F A X
$$

and hence

$$
g\left(\left(\nabla_{X} A\right) Y, U\right)+g(A F A X, Y)=(X \alpha) g(U, Y)+\alpha g(F A X, Y) .
$$


Thus

$\left.g\left(\left(\nabla_{X} A\right) Y-\nabla_{Y} A\right) X, U\right)+2 g(A F A X, Y)=(X \alpha) g(U, Y)-(Y \alpha) g(U, X)+2 \alpha g(F A X, Y)$.

This and the Codazzi equation (4.5) yield

$$
2 k(F Y, X)+2 g(A F A X, Y)=(X \alpha) u(Y)-(Y \alpha) u(X)+\alpha g((F A+A F) X, Y) .
$$

Putting $Y=U$ in (4.6) and making use of $A Y=\alpha U$, we get

$$
X \alpha=u(X) U \alpha .
$$

This, together with (4.6), implies that

$$
-2 k F X+2 A F A X=\alpha(F A+A F) X .
$$

LEMmA 4.1. Let $U$ be an eigenvector of $A$ corresponding to the eigenvalue $\alpha$ and let $X$ be the eigenvector of $A$ corresponding to the second eigenvalue $\lambda$. Then we have

$$
(2 \lambda-\alpha) A F X=(2 k+\alpha \lambda) F X .
$$

Proof. Let $X$ be an eigenvector of $A$ which corresponds to $\lambda$. Then, from (4.7), it follows that

$$
-2 k F X+2 \lambda A F X=\alpha \lambda F X+\alpha A F X,
$$

from which Lemma 4.1 follows. (See also [2], pp. 153.)

Let us consider the Levi form in this situation. From Theorem 2.1, we have

$$
L(X, Y)=\sum_{a=1}^{p}\left\{g\left(A_{a} X, Y\right)+g\left(A_{a} F X, F Y\right)\right\} \xi_{a},
$$

for $X, Y \in H(M)$. Now we note that for any $X \in H(M)=T(M) \cap J T(M)$, there exists $V \in T(M)$ such that $X=J Y=F V$ and for any $V \in T(M), F V \in H(M)$. From this fact, we may write (4.8) as follows:

$$
\begin{aligned}
L(X, Y)= & \sum_{a=1}^{p}\left\{g\left(A_{a} F V, F W\right)+g\left(A_{a} F^{2} V, F^{2} W\right)\right\} \xi_{a} \\
= & \sum_{a=1}^{p}\left\{g\left(A_{a} F V, F W\right)+g\left(A_{a} V, W\right)-u(V) g\left(A_{a} U, W\right)\right. \\
& \left.-u(W) g\left(A_{a} U, V\right)+u(V) u(W) g\left(A_{a} U, U\right)\right\} \xi_{a},
\end{aligned}
$$

where we have used (3.1).

If $\xi=\xi_{1}$ is parallel with respect to the normal connection, using relation (3.16), we can write $A_{a} U=0$, for $a=2, \ldots, p$. Thus in this case, it follows that

$$
\begin{gathered}
L(X, Y)=g(A V-F A F V-u(V) A U-u(A V) U+u(V) u(A U) U, W) \xi \\
+\sum_{a=2}^{p} g\left(A_{a} V-F A_{a} F V, W\right) \xi_{a},
\end{gathered}
$$

for any $V, W \in T(M)$.

We assume that $U$ is an eigenvector of $A$ corresponding to the eigenvalue $\alpha$ and that the Levi form vanishes at a point $x \in M$. Then from relation (4.10), we obtain

$$
A V-F A F V-\alpha u(V) U=0, \quad A_{a} V-F A_{a} F V=0,
$$


for $a=2, \ldots, p$. Let $V_{x}$ be an eigenvector of $A$ at $x$ with the eigenvalue $\lambda$, such that $V_{x}$ is orthogonal to $U$ at $x$. Then Lemma 4.1 and relation (4.11) imply that $k+\lambda^{2}=0$. Thus we have

Theorem 4.1 ([3]). Let $M$ be an $n$-dimensional $(n \geq 3)$ CR submanifold of $C R$ dimension $\frac{n-1}{2}$ of a complex space form. If the distinguished normal vector field $\xi$ to $M$ is parallel with respect to the normal connection and if $U=-J \xi$ is an eigenvector of the shape operator $A$ with respect to $\xi$, then the Levi form vanishes only when the holomorphic sectional curvature of the ambient manifold is non-positive.

TheOREM 4.2 ([3]). Let $M$ be an $n$-dimensional $(n \geq 3)$ CR submanifold of $C R$ dimension $\frac{n-1}{2}$ of a complex projective space. If $M$ satisfies the conditions of Theorem 4.1 , then the Levi form cannot vanish identically. In particular, if $M$ is a real hypersurface, then $M$ is pseudoconvex.

Since homogeneous real hypersurfaces of a complex projective space have $U$ as an eigenvector of the shape operator $A([5])$, the Levi form of a homogeneous real hypersurface of a complex projective space can never vanish. Consequently, as a special case, the Levi form of the generalized equators $M_{p, q}^{C}([9])$ and that of the geodesic spheres can never vanish.

Finally, when in $M$ the Levi form vanishes identically, $M$ is called a Levi flat submanifold. In his recent work Siu ([11]) proved that there does not exist a smooth Levi flat hypersurface of complex projective space of dimension $\geq 3$. However, if we do not assume that $M$ is complete, the following example of a real hypersurface, which is given by Kimura $([6])$, is Levi flat.

EXAMPLE 4.1. Let $\widetilde{M}$ be the hypersurface of $S^{2 n+1}$ in $\mathbf{C}^{n+1}$ given by

$$
\begin{aligned}
\widetilde{M}= & \left\{\left(r e^{\sqrt{-1} t} \cos \theta, r e^{\sqrt{-1} t} \sin \theta,\left(1-r^{2}\right)^{\frac{1}{2}} z_{2}, \ldots,\left(1-r^{2}\right)^{\frac{1}{2}} z_{n}\right) \in \mathbf{C}^{n+1},\right. \\
& \left.\sum_{j=2}^{n}\left|z_{j}\right|^{2}=1,0<r<1,0 \leq t<2 \pi \text { and } 0 \leq \theta<2 \pi\right\} .
\end{aligned}
$$

We recall that a unit sphere $S^{2 n+1}$ in $\mathbf{C}^{n+1}$ is a principal bundle with structure group $S^{1}$ and projection map $\pi$. Then $M=\pi(\widetilde{M})$ is a real hypersurface of a complex projective space. Following [6] we calculate the second fundamental form and obtain that $\widetilde{M}$ is Levi flat.

\section{References}

[1] A. Bejancu, CR-submanifolds of a Kähler manifold I, Proc. Amer. Math. Soc. 69 (1978), 135-142.

[2] M. Djorić and M. Okumura, CR submanifolds of maximal CR dimension of complex projective space, Arch. Math. 71 (1998), 148-158.

[3] M. Djorić and M. Okumura, Levi form of CR submanifolds of maximal CR dimension of complex space forms, to appear. 
[4] R. Hermann, Convexity and pseudoconvexity for complex sumanifolds, J. Math. Mech. 13 (1964), 667-672.

[5] M. Kimura, Real hypersurfaces and complex submanifolds in complex projective space, Trans. Amer. Math. Soc. 296 (1986), 137-149.

[6] M. Kimura, Sectional curvatures of holomorphic planes on a real hypersurface in $P^{n}(\mathbf{C})$, Math. Ann. 276 (1987), 487-497.

[7] W. Klingenberg, Real hypersurfaces in Kähler manifolds, SFB 288 Preprint No. 261, Differentialgeometrie und Quantenphysik, Berlin 1997.

[8] S. Kobayashi and K. Nomizu, Foundations of Differential Geometry II, Interscience, New York, 1969.

[9] H. B. Lawson, Jr., Local rigidity theorems in rank-1 symmetric spaces, J. Differential Geometry 4 (1970), 349-357.

[10] R. Nirenberg and R. O. Wells, Jr., Approximation theorems on differentiable submanifolds of a complex manifold, Trans. Amer. Math. Soc. 142 (1965), 15-35.

[11] Y.-T. Siu, Nonexistence of smooth Levi-flat hypersurfaces in complex projective spaces of dimension $\geq 3$, Ann. of Math. 151 (2000), 1217-1243. 